\title{
Accuracy criterion for the mean-field approximation in susceptible-infected-susceptible epidemics on networks
}

\author{
P. Van Mieghem* and R. van de Bovenkamp \\ Faculty of Electrical Engineering, Mathematics and Computer Science, Delft University of Technology, \\ P.O. Box 5031, 2600 GA Delft, The Netherlands \\ (Received 6 December 2014; published 30 March 2015)
}

\begin{abstract}
Mean-field approximations (MFAs) are frequently used in physics. When a process (such as an epidemic or a synchronization) on a network is approximated by MFA, a major hurdle is the determination of those graphs for which MFA is reasonably accurate. Here, we present an accuracy criterion for Markovian susceptible-infectedsusceptible (SIS) epidemics on any network, based on the spectrum of the adjacency and SIS covariance matrix. We evaluate the MFA criterion for the complete and star graphs analytically, and numerically for connected Erdős-Rényi random graphs for small size $N \leqslant 14$. The accuracy of MFA increases with average degree and with $N$. Precise simulations (up to network sizes $N=100$ ) of the MFA accuracy criterion versus $N$ for the complete graph, star, square lattice, and path graphs lead us to conjecture that the worst MFA accuracy decreases, for large $N$, proportionally to the inverse of the spectral radius of the adjacency matrix of the graph.
\end{abstract}

DOI: 10.1103/PhysRevE.91.032812

PACS number(s): $89.75 . \mathrm{Hc}$

\section{INTRODUCTION}

A particularly popular class of dynamic processes on networks can be called the local rule-global emergent properties (LrGep) class, where the collective action of the local rules executed at each node gives rise to a complex, emergent global behavior. "Local" refers to the subgraph around a node that incorporates all of the direct neighbors of that node in a graph $G$ with $N$ nodes and $L$ links, and sometimes also all links between those direct neighbors. Some examples of the LrGep class are epidemic models [such as susceptibleinfected-susceptible (SIS) and susceptible-infected-recovered (SIR)] and more general reaction-diffusion processes [1], the Ising spin model [2], the Kuramoto coupled-oscillator model [3], sand piles as models for self-organized criticality [4-6], and opinion models $[7,8]$. Many LrGep models feature, in general, a phase transition [9]; they all depend heavily on the underlying network topology and many processes in nature seem well described by LrGep models.

The simplicity of the local rules disguises the overwhelming, fascinating complexity of the global emergent behavior in the network that these local rules create. The complexity generated by the simple local rules is readily understood by concentrating on the SIS epidemic model [10-13], where each node $j$ at time $t$ can either be in an infected state $\left[X_{j}(t)=1\right]$ or in a healthy state $\left[X_{j}(t)=0\right]$. The SIS local rule consists of two possible actions: (a) an infected node can infect its healthy neighbors and (b) an infected node can cure and thereafter become again susceptible to the infection through its infected neighbors. When assuming a Poisson infection process with rate $\beta$ and a Poisson curing process with rate $\delta$, the local nodal dynamics is described by a two-state Markov process in continuous time, which is analytically solvable in explicit form [14]. In a connected network, node $j$ has $d_{j}$ neighbors, and each of its neighbors can also be either infected or healthy. Moreover, the network connectivity enables infection spread

\footnotetext{
*p.f.a.vanmieghem@tudelft.nl
}

over links, thereby coupling the local state of node $i$ and node $j$. In particular, the local infection rate $\beta$ of node $i$ is now replaced by the network infection rate $\beta \sum_{j \in \text { neighbor }(i)} X_{j}(t)$ because node $i$ can be infected along its links by all of its infected neighbors. After coupling all local two-state Markovian processes, a global SIS Markovian process on the network with $N$ nodes originates, which now consists of $2^{N}$ states, covering all possible combinations in which $N$ nodes can be infected $[15,16]$. Suddenly, the simple two-state process explodes to a complex $2^{N}$-state Markovian process. A general theorem (arising from linear system theory) states that any dynamic process can be approximated arbitrarily close by a Markovian process with sufficiently large state space. Hence, Markovian processes with a large state space can mimic general dynamic processes, which may explain the potential of rich and complex behavior (such as phase transitions) in LrGep models.

Even for the simple SIS model, where the network coupling $\beta \sum_{j \in \text { neighbor }(i)} X_{j}(t)$ is linear in the state variables $\left\{X_{j}\right\}_{1 \leqslant j \leqslant N}$ in contrast to most other LrGep models, very few exact solutions exist [17-19]. The difficulty in finding exact solutions [20] has spurred the search for approximations. One of the most successful approximations is the so-called mean-field approximation (MFA). The MFA originates from many-body interacting particle systems [21], in which each (charged) particlemost often an electron-generates an electric Coulomb field, but experiences the resultant field from all other particles. By replacing the actual resultant field by the mean electric field, the many-body governing equations greatly simplify, basically to the one-electron Schrödinger equation [22,23], whose solution was shown to describe reality surprisingly well.

\section{MEAN-FIELD APPROXIMATION FOR MARKOVIAN SIS EPIDEMICS ON NETWORKS}

The MFA, applied to SIS epidemics on networks, replaces the actual infection rate $\beta \sum_{k=1}^{N} a_{k i} X_{k}=\beta \sum_{j \in \text { neighbor }(i)} X_{j}(t)$ towards node $i$ by its average rate $\beta \sum_{k=1}^{N} a_{k i} E\left[\bar{X}_{k}(t)\right]$, where $\overline{X_{k}}$ refers to the MFA state of node $k$ and $a_{k i}$ is an element 
of the adjacency matrix $A$ of the graph $G$. If the neighboring states are sufficiently weakly dependent and the number of neighbors $d_{i}$ (degree of node $i$ ) is huge so that the Central Limit Theorem [14] is applicable, then the replacement of a random variable by its mean is increasingly accurate for growing $d_{i}$. The mathematical conditions for the validity of the Central Limit Theorem, stated and proved by Lindeberg [24], translate, more or less, to the physical interpretation that "fluctuations" (i.e., the variance of all $X_{i}$ ) are small and none of them is dominant in the "thermodynamic limit" (i.e., $N \rightarrow \infty)$. Thus, MFA implicitly assumes "large degrees" in graphs when the graph size $N$ grows. The latter hints that MFA is likely not accurate (i) in lattices or regular graphs with fixed degree $r$, not depending on $N$, as confirmed by simulations in, e.g., [25], and (ii) in small graphs, say $N<10$.

Another consequence of replacing the random variable by its mean in MFAs results in the neglect of correlations in the dynamic process: MFA treats the joint probability $\operatorname{Pr}\left[X_{i}=1, X_{j}=1\right]$ as if $X_{i}$ and $X_{j}$ were independent, $\operatorname{Pr}\left[X_{i}=1\right] \operatorname{Pr}\left[X_{j}=1\right]$. We elaborate on the dependence by considering the exact Markovian SIS governing equation $[14,26]$ for the infection probability of node $i$,

$$
\begin{aligned}
\frac{d E\left[X_{i}\right]}{d t}= & E\left[-\delta X_{i}+\beta\left(1-X_{i}\right) \sum_{k=1}^{N} a_{k i} X_{k}\right] \\
= & -\delta E\left[X_{i}\right]+\beta \sum_{k=1}^{N} a_{k i} E\left[X_{k}\right] \\
& -\beta \sum_{k=1}^{N} a_{k i} E\left[X_{i} X_{k}\right] .
\end{aligned}
$$

The time derivative of the infection probability $E\left[X_{i}\right]=$ $\operatorname{Pr}\left[X_{i}=1\right]$ of a node $i$ consists of the expectation of two competing processes in (1), expressed in the Bernoulli random variable $X_{i} \in\{0,1\}$ : (i) while node $i$ is healthy, i.e., not infected $\left(1-X_{i}\right)$, all infected neighbors $\sum_{k=1}^{N} a_{k i} X_{k}$ of node $i$ try to infect the node $i$ with rate $\beta$, and (ii) while node $i$ is infected $X_{i}$, the node $i$ is cured at rate $\delta$. Introducing the covariance $\left[14\right.$, p. 25], $\operatorname{cov}\left[X_{i}, X_{k}\right]=E\left[X_{i} X_{k}\right]-E\left[X_{i}\right] E\left[X_{k}\right]$, leads to

$$
\begin{aligned}
\frac{d E\left[X_{i}\right]}{d t}= & -\delta E\left[X_{i}\right]+\beta\left(1-E\left[X_{i}\right]\right) \sum_{k=1}^{N} a_{k i} E\left[X_{k}\right] \\
& -\beta \sum_{k=1}^{N} a_{k i} \operatorname{cov}\left[X_{i} X_{k}\right]
\end{aligned}
$$

Finally, in terms of the infection probability of node $i, w_{i}(t)=$ $\operatorname{Pr}\left[X_{i}(t)=1\right]=E\left[X_{i}(t)\right]$, we have

$$
\begin{aligned}
\frac{d w_{i}(t)}{d t}= & -\delta w_{i}(t)+\beta\left[1-w_{i}(t)\right] \sum_{k=1}^{N} a_{k i} w_{k}(t) \\
& -\beta \sum_{k=1}^{N} a_{k i} \operatorname{cov}\left[X_{i}(t), X_{k}(t)\right],
\end{aligned}
$$

from which we recognize the first part as the $N$-intertwined mean-field approximation (NIMFA) equation [16] in the mean- field infection probability $v_{i}(t)=\operatorname{Pr}\left[\bar{X}_{i}(t)=1\right]$ of node $i$,

$$
\frac{d v_{i}(t)}{d t}=-\delta v_{i}(t)+\beta\left[1-v_{i}(t)\right] \sum_{k=1}^{N} a_{k i} v_{k}(t) .
$$

For each node $i$, we may consider $\beta R_{i}$ in (2), where

$$
R_{i}=\sum_{k=1}^{N} a_{k i} \operatorname{cov}\left[X_{i}(t), X_{k}(t)\right]
$$

as the MFA correction term, whose omission in (2) specifies the impact or accuracy (per node) of MFA. Clearly, if $\operatorname{cov}\left[X_{i}(t), X_{k}(t)\right]=0$ for each nodal pair $(i, k)$, then the NIMFA equations (3) are equal to the exact SIS equations (2). Moreover, as shown in [27], $\operatorname{cov}\left[X_{i}(t), X_{k}(t)\right] \geqslant 0$ for a Markovian SIS and SIR process on any graph, so that $\beta R_{i} \geqslant 0$ and NIMFA always upper bounds the viral infection probability $\left(v_{i} \geqslant w_{i}\right)$ and, thus, lower bounds the epidemic threshold $\tau_{c} \geqslant \tau_{c}^{(1)}=\frac{1}{\lambda_{1}}$, where $\lambda_{1}$ is the spectral radius of the adjacency matrix $A$ of the graph [28] and $\tau=\frac{\beta}{\delta}$ is the effective infection rate.

The effect of MFA $[15,16]$ is that the $2^{N}$ linear Markovian equations in the joint probabilities of the set $\left\{X_{j}(t)\right\}_{1 \leqslant j \leqslant N}$ are replaced by $N$ nonlinear equations (3) in the nodal infection probabilities $\operatorname{Pr}\left[\bar{X}_{j}(t)=1\right]$. In addition, although nonlinear, these $N$ MFA equations, which characterize the $N$-intertwined mean-field approximation [16], allow us to deduce more insight and often lead to sufficiently accurate results, in particular, in regimes far enough away from the phase transition and for large graphs. As a result, many papers even start a discussion on SIS epidemics from their MFA equations, completely ignoring the approximate character. However, for some large graphs ${ }^{1}$ and certain parameter sets close to the phase transition (specified by the epidemic threshold $\tau_{c}$ ), MFA can be unacceptably inaccurate [1]. A fundamental open problem is the determination of a criterion for graphs, akin to a Ginzburg-like criterion, ${ }^{2}$ which specifies the accuracy of MFA as a function of the graph. In particular, for SIS epidemics described by (2), this question translates to "which graph (or set of graphs) minimizes $R_{i}$ in (4) for any node $i$ ?" So far (e.g., [25]), the accuracy of MFA has been evaluated by comparing the MFA epidemic threshold $\tau_{c}^{(1)}$ (or metastable state fraction of infected nodes) with the exact SIS epidemic threshold $\tau_{c}$. Unfortunately, the epidemic threshold as an accuracy metric is difficult to compute precisely and does not provide much insight to determine for which graph MFA is accurate. In the remainder, we propose another accuracy criterion based on $R_{i}$ in (4).

\footnotetext{
${ }^{1}$ For small graphs with $N<10$ nodes, the MFA in SIS epidemics is, in most cases, inadequate. However, in those cases, the $2^{N}$ linear Markov equations can be solved numerically [17].

${ }^{2}$ The Ginzburg criterion, discussed in [29], assesses the validity of mean-field theory in physical systems. However, that criterion does not straightforwardly translate to conditions on the underlying graphs over which the dynamic process runs.
} 


\section{ACCURACY CRITERION}

Let $C$ denote the symmetric covariance matrix, with $c_{i j}=$ $\operatorname{cov}\left[X_{i}, X_{j}\right]=c_{j i}$, so that (4) equals $R_{i}=\sum_{k=1}^{N} a_{i k} c_{k i}$. As demonstrated in [27], $c_{i j} \geqslant 0$, and, since $a_{i j} \geqslant 0$, we have $R_{i} \geqslant 0$ for each node $i$. Let $R, V, W$ be the vector with $i$ th component $R_{i}, v_{i}(t)$, and $w_{i}(t)$, respectively. The SIS vector differential equation follows from (2) and (4) as

$$
\frac{d W(t)}{d t}=-\delta W(t)+\beta \operatorname{diag}\left[1-w_{i}(t)\right] A W(t)-\beta R .
$$

The MFA minimization condition, i.e., $R_{i}$ for each $i$, translates to the minimization of a vector norm for $R$ such as the Hölder $q$ norm [14], $\|R\|_{q}=\left(\sum_{i=1}^{N} R_{i}^{q}\right)^{1 / q}$ for real $q>0$. Since $\|R\|_{1}(q=1)$ has a simple analytic expression, we propose to determine the accuracy of MFA for a graph $G$ by

$\|R\|_{1}=\sum_{i=1}^{N}\left(\sum_{k=1}^{N} a_{i k} c_{k i}\right)=\operatorname{tr}(A C)=\operatorname{tr}(C A)=\sum_{k=1}^{N} \lambda_{k}(A C)$,

where $\lambda_{1}(M) \geqslant \lambda_{2}(M) \geqslant \cdots \geqslant \lambda_{N}(M)$ are the ordered real eigenvalues of an $N \times N$ symmetric matrix $M$. We remark that criterion (6) does not hold for non-Markovian SIS epidemics on networks [30], since then, as shown in [27], $c_{i j}=$ $\operatorname{cov}\left[X_{i}, X_{j}\right]$ can be negative. A consequence of the WielandtHoffman theorem [28, p. 252] for symmetric matrices $A$ and $C$ is that

$$
\|R\|_{1}=\sum_{k=1}^{N} \lambda_{k}(A C) \leqslant \sum_{k=1}^{N} \lambda_{k}(A) \lambda_{k}(C) .
$$

Hence, (7) demonstrates that the minimum of $\|R\|_{1}$ is achieved when the eigenvalue vectors of $A$ and $C$ are as orthogonal as possible to each other. The upper bound, derived in Appendix A,

$$
\sum_{k=1}^{N} \lambda_{k}(A) \lambda_{k}(C) \leqslant \frac{E_{G}}{2}\left[\lambda_{1}(C)-\lambda_{N}(C)\right],
$$

where the graph energy [28, p. 201] is defined as $E_{G}=$ $\sum_{k=1}^{N}\left|\lambda_{k}(A)\right|$, is attainable for some graphs (such as the complete graph and star graph, as shown below).

The analysis of the spectrum of $C$ in Appendix B indicates that $\lambda_{1}(C)$ is dominant, while all other $\lambda_{k}(C) \geqslant 0$ are relatively small, which suggests that we write (7) as

$$
\begin{aligned}
\sum_{k=1}^{N} \lambda_{k}(A) \lambda_{k}(C)= & \lambda_{1}(A) \lambda_{1}(C)+\sum_{k=2}^{l} \lambda_{k}(A) \lambda_{k}(C) \\
& +\sum_{k=l+1}^{N} \lambda_{k}(A) \lambda_{k}(C),
\end{aligned}
$$

where the index $l$ is such that $\lambda_{l}(A)>0$ and $\lambda_{l+1}(A) \leqslant 0$. The first term $\lambda_{1}(A) \lambda_{1}(C)$ is large and dominant, while the last sum is negative. The minimization of the left-hand side in (9) requires that $l$ is as small as possible and the last sum is, in absolute value, as large as possible.

Suppose that $\lambda_{k}(C)=\lambda_{C}$ for all $k$; then it follows from (7) that $\|R\|_{1}=0$ because [28], for any graph, $\sum_{k=1}^{N} \lambda_{k}(A)=0$. However, when $\lambda_{k}(C)=\lambda_{C}$ for all $k$, then $C=\lambda_{C} I$ because any real symmetric matrix $M$ has the eigenvalue decomposition $M=U \operatorname{diag}\left[\lambda_{k}(M)\right] U^{T}$, where $U$ is an orthogonal matrix satisfying $U^{T} U=U U^{T}=I$. Hence, when $C=\lambda_{C} I$, it holds that $c_{i k}=\operatorname{cov}\left[X_{i}(t), X_{k}(t)\right]=0$ for any $k \neq i$, which, indeed, would imply that MFA is exact. However, such a covariance matrix does not exist because $c_{i j}>0$ for an arbitrary effective infection rate $\tau$, even for the complete graph $K_{N}$. Thus, MFA cannot be exact so that $\|R\|_{1}>0$.

\section{NORMALIZED CRITERION}

So far, $\|R\|_{1}$ has been discussed as an absolute measure. We present two normalization criteria $r$, i.e., one that is purely topological and one related to the SIS process, with the property $0 \leqslant r \leqslant 1$. If $r=0$ for a graph with a given effective infection rate $\tau$, then MFA is exact, whereas for $r=1$, MFA provides the worst possible accuracy among all graphs.

From definition (6) and $0 \leqslant c_{i j} \leqslant \frac{1}{4}$ (see Appendix B), it follows directly that

$$
\|R\|_{1}=\sum_{i=1}^{N} \sum_{k=1}^{N} a_{i k} c_{k i} \leqslant \frac{1}{4} \sum_{i=1}^{N} \sum_{k=1}^{N} a_{i k}=\frac{L}{2},
$$

where $L$ is the number of links in graph $G$. The normalized "graph topology" criterion is

$$
r_{T}=\frac{2\|R\|_{1}}{L} .
$$

The SIS vector differential equation (5) indicates that the norm $\|R\|_{1}$ could be compared with

$$
\begin{aligned}
\left\|\operatorname{diag}\left[1-w_{i}(t)\right] A W(t)\right\|_{1} & =\sum_{i=1}^{N} \sum_{k=1}^{N}\left(1-w_{i}\right) a_{i k} w_{k} \\
& =(u-W)^{T} A W,
\end{aligned}
$$

where $u=(1,1, \ldots, 1)$ is the $N \times 1$ all-one vector. Since

$$
\begin{aligned}
E\left[X_{i} X_{k}\right]= & \operatorname{Pr}\left[X_{i}=1, X_{k}=1\right]=\operatorname{Pr}\left[X_{i}=1 \mid X_{k}=1\right] \\
& \times \operatorname{Pr}\left[X_{k}=1\right] \leqslant \operatorname{Pr}\left[X_{k}=1\right]=E\left[X_{k}\right],
\end{aligned}
$$

we observe that, in (1),

$$
\sum_{k=1}^{N} a_{k i} E\left[X_{i} X_{k}\right] \leqslant \sum_{k=1}^{N} a_{k i} E\left[X_{k}\right],
$$

from which, for each node $i$,

$$
R_{i} \leqslant\left(1-E\left[X_{i}\right]\right) \sum_{k=1}^{N} a_{k i} E\left[X_{k}\right],
$$

so that, after summing over all nodes,

$$
0 \leqslant\|R\|_{1} \leqslant\left\|\operatorname{diag}\left[1-w_{i}(t)\right] A W(t)\right\|_{1} .
$$


The steady-state NIMFA ${ }^{3}$ average fraction of infected nodes [14, p. 467] obeys for $\tau>\tau_{c}^{(1)}$, as a consequence of $c_{i j} \geqslant 0$,

$$
y_{\infty}^{(1)}(\tau)=\frac{\tau}{N}\left[\left(u-V_{\infty}\right)^{T} A V_{\infty}\right] \geqslant \frac{\tau}{N}\left[\left(u-W_{\infty}\right)^{T} A W_{\infty}\right],
$$

leading to

$$
\|R\|_{1} \leqslant\left\|\operatorname{diag}\left[1-w_{i}(t)\right] A W(t)\right\|_{1} \leqslant \frac{N y_{\infty}^{(1)}(\tau)}{\tau},
$$

which leads us to propose, for $\tau \geqslant \tau_{c}>\tau_{c}^{(1)}=\frac{1}{\lambda_{1}(A)}$, the normalized "process" criterion

$$
r_{P}=\frac{\tau\|R\|_{1}}{N y_{\infty}^{(1)}(\tau)}
$$

where $\|R\|_{1}$ must be evaluated in the metastable state (which corresponds to the NIMFA steady state). Since $r \leqslant 1$, it follows that, when $\tau \downarrow \tau_{c}^{(1)}$, then $\|R\|_{1}=O\left[\left(\tau-\tau_{c}^{(1)}\right)^{1+\epsilon}\right]$ with $\epsilon>0$ (see [31]), while $\left\|^{4}\right\| R \|_{1}=o\left(\tau^{-1}\right)$ when $\tau \rightarrow \infty$.

\section{EXAMPLES}

We apply the above theory to two graphs, i.e., the complete graph $K_{N}$ and the star graph $K_{1, N-1}$, for which both the exact SIS [18] and NIMFA [15,16] are analytically soluble, as well as the spectrum of the adjacency matrix $A$ and the covariance matrix $C$ (in the metastable state). Moreover, a study [33] on the average extinction (or virus survival) time seems to hint that for Markovian SIS epidemics, $K_{N}$ and $K_{1, N-1}$ are two extremes among all graphs.

\section{A. Compete graph $K_{N}$}

Only in the complete graph is $l=1$ in (9) and the second largest eigenvalue $\lambda_{2}\left(A_{K_{N}}\right)=-1$, while in all other graphs, $l \geqslant 2$, i.e., $\lambda_{2}(A)>0$. For $K_{N}$, we find from (9) that

$$
\begin{aligned}
\sum_{k=1}^{N} \lambda_{k}\left(A_{K_{N}}\right) \lambda_{k}\left(C_{K_{N}}\right) & =(N-1) \lambda_{1}\left(C_{K_{N}}\right)-\sum_{k=2}^{N} \lambda_{k}\left(C_{K_{N}}\right) \\
& =N \lambda_{1}\left(C_{K_{N}}\right)-\sum_{k=1}^{N} \operatorname{var}\left[X_{k}\right] \\
& >N\left[\lambda_{1}\left(C_{K_{N}}\right)-\frac{1}{4}\right] .
\end{aligned}
$$

In the metastable state in $K_{N}$, we have, due to symmetry, $C_{\infty}=$ $\gamma J+\left(\sigma_{X}^{2}-\gamma\right) I$, where $\gamma=\operatorname{cov}\left[X_{i}, X_{j}\right]=c_{12}$ for $i \neq j$ is a function of the effective infection rate $\tau$, as shown in Fig. 1,

\footnotetext{
${ }^{3}$ Since NIMFA is a first order mean-field approximation [26], we write the relevant NIMFA quantities with a superscript (1), such as the NIMFA epidemic threshold $\tau_{c}^{(1)}$ and NIMFA average fraction of infected nodes $y^{(1)}$. Evaluation in the steady state (where the time $t \rightarrow \infty)$ is marked by the subscript $\infty$, such as $y_{\infty}=\lim _{t \rightarrow \infty} y(t)$.

${ }^{4}$ This follows from the Laurent series [32] [14, p. 466] for the metastable infection probability $v_{i \infty}(\tau)$ of node $i$.
}

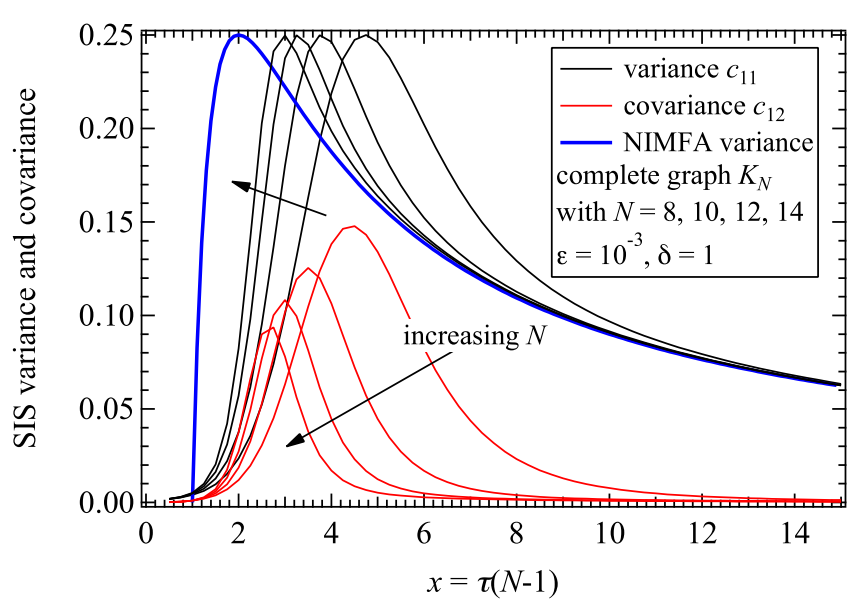

FIG. 1. (Color online) The variance $c_{i i}=\operatorname{var}\left[X_{i}\right]=c_{11}$ and covariance $c_{i j}=\operatorname{cov}\left[X_{i}, X_{j}\right]=c_{12}$ of Markovian SIS epidemics on the complete graph $K_{N}$ vs the normalized infection rate $x=\frac{\tau}{\tau_{c}^{(1)}}$ for sizes $N=8,10,12$, and 14 . Both the variance and covariance in the metastable state are computed from the exact Markov chain as explained in [17] for $\varepsilon=10^{-3}$. Also, the NIMFA variance $\left(1-\frac{1}{x}\right) \frac{1}{x}$ is shown (thick blue line).

and $J$ is the all-one matrix. Using $J^{2}=N J$,

$$
\begin{aligned}
\|R\|_{1} & =\operatorname{tr}\left(A_{K_{N}} C_{\infty}\right)=\operatorname{tr}\left\{(J-I)\left[c J+\left(\sigma_{X}-c\right) I\right]\right\} \\
& =2\left(\begin{array}{c}
N \\
2
\end{array}\right) \gamma .
\end{aligned}
$$

The eigenvalues of $C_{\infty}=\gamma J+\left(\sigma_{X}^{2}-c\right) I$ are $\lambda_{1}\left(C_{K_{N}}\right)=$ $\sigma_{X}^{2}+\gamma(N-1)$ and $\lambda_{k}\left(C_{K_{N}}\right)=\sigma_{X}^{2}-\gamma$ for $k>1$, so that

$$
\sum_{k=1}^{N} \lambda_{k}\left(A_{K_{N}}\right) \lambda_{k}\left(C_{\infty}\right)=\operatorname{tr}\left(A_{K_{N}} C_{\infty}\right),
$$

illustrating that equality holds in the Wielandt-Hoffman bound (7). The graph energy of $K_{N}$ is $E_{G}=2(N-1)$ and (8) equals

$$
\frac{E_{G}}{2}\left[\lambda_{1}\left(C_{\infty}\right)-\lambda_{N}\left(C_{\infty}\right)\right]=\gamma N(N-1)=\operatorname{tr}\left(A_{K_{N}} C_{\infty}\right),
$$

which indicates that the upper bound (8) is attained in the metastable state. The normalized "graph topology" criterion (10) is $r_{T}\left(K_{N}\right)=4 \gamma$. Finally, the normalized "process" criterion (11) equals, for $\tau>\frac{1}{N-1}$,

$$
r_{P}\left(K_{N}\right)=\gamma \frac{[(N-1) \tau]^{2}}{[(N-1) \tau-1]} .
$$

Using the normalization $x_{K_{N}}=(N-1) \tau=\frac{\tau}{\tau_{c ; K_{N}}^{(1)}}$ and $r_{T}\left(K_{N}\right)=4 \gamma$ (which is a function of $x_{K_{N}}$ ), we finally arrive at

$$
r_{P}\left(K_{N}\right)=r_{T}\left(K_{N}\right) \frac{x_{K_{N}}^{2}}{4\left(x_{K_{N}}-1\right)},
$$

which is valid for $x_{K_{N}}>1$. The largest inaccuracy in $r_{P}\left(K_{N}\right)$ seems to occur around the NIMFA epidemic threshold (due to the pole at $x_{K_{N}}=1$ ), but $\lim _{x_{K_{N}} \rightarrow 1} \frac{r_{T}\left(K_{N}\right)}{x_{K_{N}}-1}=0$ (as explained above) and $r_{T}\left(K_{N}\right)$ decays for increasing $x_{K_{N}}$ [because $\gamma=$ $o(x)$ when $x \rightarrow \infty$ ].

Both $r_{T}\left(K_{N}\right)$ and $r_{P}\left(K_{N}\right)$ underline the importance of the covariance $\gamma=\operatorname{cov}\left[X_{i}, X_{j}\right]$, whose decreasing magnitude 
with $N$ is shown in Fig. 1. Figure 1 also exhibits that the maximum variance of $\frac{1}{4}$ is reached for any graph, around that value of $\tau$ for which $y_{\infty}^{(1)}(\tau)=\frac{1}{2}$.

\section{B. Star graph $K_{1, N-1}$}

The adjacency eigenvalues of the star with $N$ nodes are $\lambda_{1}\left(A_{\text {star }}\right)=-\lambda_{N}\left(A_{\text {star }}\right)=\sqrt{N-1}$, while all others are zero. The structure of the covariance matrix $C_{\text {star }}$ in the metastable state is given in Appendix $\mathrm{C}$, from which

$$
\|R\|_{1}=\operatorname{tr}\left(A_{\text {star }} C_{\text {star }}\right)=2(N-1) c,
$$

where $c$ is the SIS covariance of the center and a leaf node. All covariances are bell-shaped functions of $\tau$, as illustrated in Fig. 2. The normalized graph topology criterion (10) becomes

$$
r_{T}\left(K_{1, N-1}\right)=4 c .
$$

The Wielandt-Hoffman bound (9),

$$
\sum_{k=1}^{N} \lambda_{k}\left(A_{\text {star }}\right) \lambda_{k}\left(C_{\text {star }}\right)=\sqrt{N-1}\left[\lambda_{1}\left(C_{\text {star }}\right)-\lambda_{N}\left(C_{\text {star }}\right)\right]
$$

shows, for any effective infection rate $\tau$, that the upper bound (8) is also attained for the star graph. The normalized graph topology criterion (10) is upper bounded by

$$
r_{T}\left(K_{1, N-1}\right) \leqslant 2 \frac{\left[\lambda_{1}\left(C_{\text {star }}\right)-\lambda_{N}\left(C_{\text {star }}\right)\right]}{\sqrt{N-1}},
$$

and, explicitly, using the spectrum computed in Appendix C,

$$
r_{T}\left(K_{1, N-1}\right) \leqslant 2 \sqrt{\left(\frac{b-a}{\sqrt{N-1}}+\sqrt{N-1} d\right)^{2}+4 c^{2}} .
$$

For large $N$,

$$
r_{T}\left(K_{1, N-1}\right) \sim 2 \sqrt{N} d,
$$

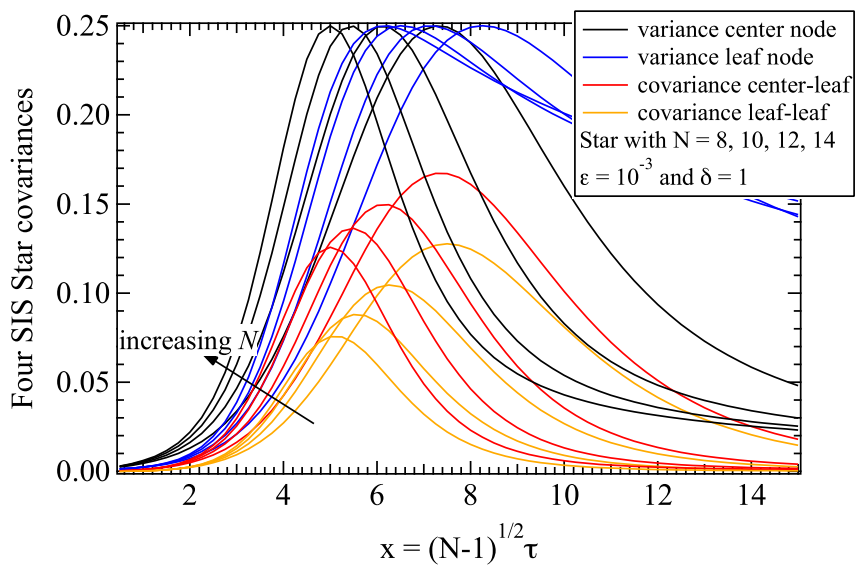

FIG. 2. (Color online) The variance of the center node $c_{11}=a$ and of the leaf node $c_{22}=b$ and the covariance of the center leaf $c_{12}=c$ and of the leaf leaf $c_{23}=d$ (see Appendix C) of Markovian SIS epidemics on the star graph $K_{1, N}$ vs the normalized infection rate $x=\frac{\tau}{\tau_{c}^{(1)}}$ for sizes $N=8,10,12,14$ and $\varepsilon=10^{-3}$. The computation is based on the solution of the $2^{N} \times 2^{N}$ exact Markov infinitesimal generator.

where $d$ is the covariance between leaf nodes, which is bounded from below by (C3). In contrast to the complete graph, (9) upper bounds $\operatorname{tr}\left(A_{\text {star }} C_{\text {star }}\right)$. Simulations (see Fig. 3 below) suggest that $c=O\left(\frac{1}{\sqrt{N}}\right)$ for large $N$ and that the Wielandt-Hoffman bound (9) for $\operatorname{tr}\left(A_{\text {star }} C_{\text {star }}\right)$ is accurate for large $N$, so that $2 \sqrt{N} d \sim 4 c$. In that case, the inequality (C3) indeed indicates that $c=O\left(\frac{1}{\sqrt{N}}\right)$ and $d=O\left(\frac{1}{N}\right)$. Hence, we expect that $r_{T}\left(K_{1, N-1}\right) \sim O\left(\frac{1}{\sqrt{N}}\right)$ for large $N$.

Using $y_{\infty, \text { star }}^{(1)}(\tau)=\frac{\left(N-1-\frac{1}{\tau^{2}}\right)}{N}\left(\frac{1}{\frac{1}{\tau}+1}+\frac{1}{\frac{1}{\tau}+N-1}\right) \quad$ (see, e.g., [15]), the normalized process criterion (11) equals, for $\tau>$ $\frac{1}{\sqrt{N-1}}$,

$$
r_{P}\left(K_{1, N-1}\right)=\frac{\tau \sqrt{N-1}\left[\lambda_{1}\left(C_{\text {star }}\right)-\lambda_{N}\left(C_{\text {star }}\right)\right]}{\left(N-1-\frac{1}{\tau^{2}}\right)\left(\frac{1}{\frac{1}{\tau}+1}+\frac{1}{\frac{1}{\tau}+N-1}\right)}=\frac{x_{\text {star }}}{\left(x_{\text {star }}^{2}-1\right)} \frac{\frac{x_{\text {star }}}{\sqrt{N-1}}\left(1+\frac{x_{\text {star }}}{\sqrt{N-1}}\right)\left(1+x_{\mathrm{star}} \sqrt{N-1}\right)\left[\lambda_{1}\left(C_{\text {star }}\right)-\lambda_{N}\left(C_{\text {star }}\right)\right]}{2+\frac{N}{\sqrt{N-1}} x_{\text {star }}},
$$

where $x_{\text {star }}=\tau \sqrt{N-1}=\frac{\tau}{\tau_{c \text { star }}^{\text {(1) }}}>1$. If $N$ is large, $x_{\text {star }}$ does not dependent on $N$ and $x_{\text {star }}>1$, then

$$
r_{P}\left(K_{1, N-1}\right) \sim \frac{\left[\lambda_{1}\left(C_{\mathrm{star}}\right)-\lambda_{N}\left(C_{\mathrm{star}}\right)\right]}{\sqrt{N-1}} \frac{x_{\mathrm{star}}^{2}}{\left(x_{\mathrm{star}}-1\right)\left(x_{\mathrm{star}}+1\right)}=r_{T}\left(K_{1, N-1}\right) \frac{x_{\mathrm{star}}^{2}}{2\left(x_{\mathrm{star}}-1\right)\left(x_{\mathrm{star}}+1\right)},
$$

illustrating that around the NIMFA epidemic threshold $\left(x_{\text {star }}=1\right)$, the normalized criterion $r_{P}$ behaves for the star graph similarly as for the complete graph. The prefactor $r_{T}$, namely, $\gamma$ versus $c$, makes the difference.

Both examples indicate that we can further confine ourselves to the simpler normalized graph topology criterion $r_{T}$ in (10) and that the correlation matrix $C$, which depends on $\tau$, is key. The bell-shaped nature of the covariances $\operatorname{cov}\left[X_{i}, X_{j}\right]$ as a function of $\tau$ shows that for any graph, there is an effective infection rate $\tau$ for which the normalized criterion $r_{T}$ is largest, corresponding to the worst MFA accuracy for that graph. We propose to use $\max _{\tau} r_{T}$ for the comparison. Other choices, such as $\int_{0}^{\infty} r_{T}(s) d s$, where $s=\frac{1}{\tau}$, inspired by the viral conductance
[32,34,35], are possible, though more complicated to evaluate. The examples above suggest that the maximum value $\max _{\tau} r_{T}$ is reached at an effective infection rate $\tau<\tau^{*}$ slightly smaller than the solution of $\frac{1}{2}=y_{\infty}^{(1)}\left(\tau^{*}\right)$ (which maximizes the average of the variances over all nodes) and $\tau^{*}<5 \tau_{c}^{(1)}$ for $N>14$.

\section{Scaling of $\max _{\tau} r_{T}$ with the network size $N$}

Figure 3 shows the scaling of $\max _{\tau} r_{T}$ versus the network size $N$ for the complete graph $K_{N}$, the path graph $P_{N}$ (a tree 


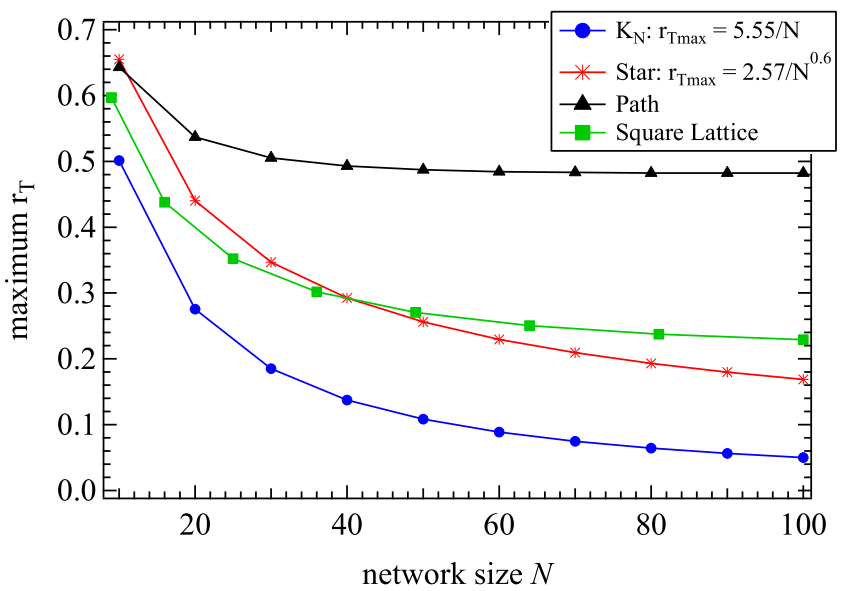

FIG. 3. (Color online) The scaling of $\max _{\tau} r_{T}$ vs the network size $N$ for the complete graph $K_{N}$, the path graph, and the star graph $K_{1, N-1}$, for $N$ ranging from $N=10$ up to $N=100$.

with maximum hop count $N-1$ ), the star graph $K_{1, N-1}$, and the square lattice, for $N$ ranging from $N=10$ up to $N=100$. The data were obtained by long simulations, described in detail in [25]. The simulations for $N=10$ were compared to the $2^{N}$-state Markov chain solution and were more accurate than two significant digits, so that the simulations were indistinguishable from the Markov chain solution in a plot.

On the interval $[10,100]$, fitting the data revealed that

$$
\max _{\tau} r_{T}\left(K_{N}\right) \simeq \frac{5.55}{N}
$$

while

$$
\max _{\tau} r_{T}\left(K_{1, N-1}\right) \simeq \frac{2.57}{N^{0.6}}
$$

For large $N$, we expected in the star graph, as explained in Sec. VB, a scaling as $O\left(\frac{1}{\sqrt{N}}\right)$. Exact computations of the star graph in [18] indicate a rather slow tendency of the star graph towards its asymptotic SIS regime, so that much larger network sizes than $N=100$ need to be considered to enter the asymptotic regime. Figure 3 also shows that the path graph has the worst MFA accuracy: $\max _{\tau} r_{T}\left(P_{N}\right)$ remains constant from $N>60$ on, which hints that $\max _{\tau} r_{T}\left(P_{N}\right)$ tends to a constant for large $N$. The MFA accuracy of the square lattice, $\max _{\tau} r_{T}(\mathrm{La})$, does not decrease as $O\left(N^{-a}\right)$ for some positive real number $a$, but tends to flatten for high $N$. We speculate that $\max _{\tau} r_{T}(\mathrm{La})$, similar as for the path graph, also tends towards a constant for $N>100$.

\section{Influence of the average degree on $\boldsymbol{r}_{T}$}

The normalized topological MFA criterion $r_{T}$ is computed, after solving the steady-state of the exact $\varepsilon$-SIS Markov chain for a self-infection rate $\varepsilon=10^{-3}$, which we define as the metastable state in Markovian SIS epidemics on finite networks $[14,17,18]$. Since these computations are limited to small graphs $N \leqslant 14$ (due to the $2^{N}$-state space of the SIS Markov chain), we concentrated on connected Erdős-Rényi random graphs $G_{r}(N, L)$ with precisely $N$ nodes and precisely $L$ links [14, Sec. 15.7]. For these small sizes, the class $G_{r}(N, L)$ samples sufficiently over all possible graphs. Characteristic

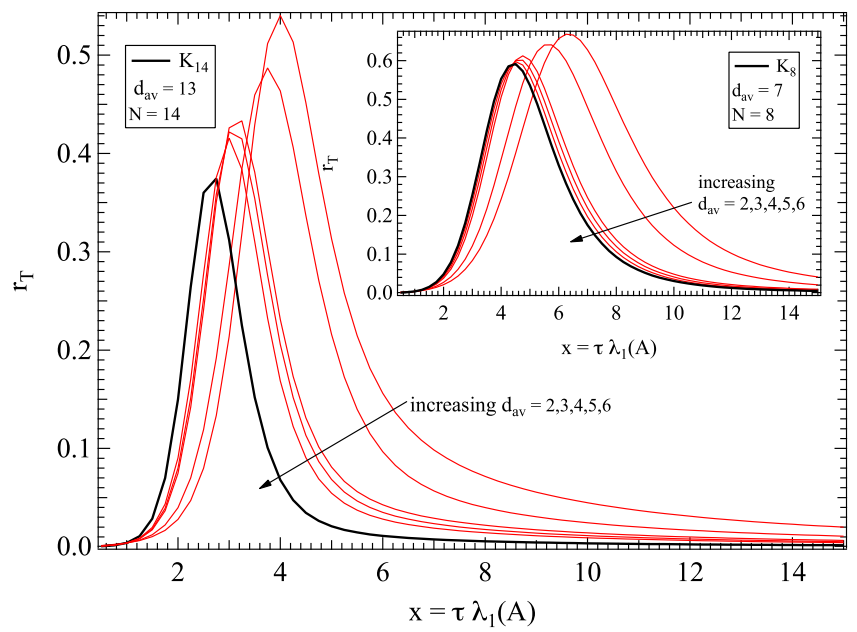

FIG. 4. (Color online) The normalized topological MFA criterion $r_{T}$ vs the normalized effective infection rate $x=\tau \lambda_{1}(A)$ for Erdôs-Rényi random graphs $G_{r}(N, L)$ with $N=14$ (Inset: $N=8$ ) and average degrees $d_{\mathrm{av}}$ varying from 2 to $6 ; r_{T}$ for the complete graph $K_{N}$ is also shown (in thick black line).

graph properties, such as scale freeness [36], are only clearly observed from $N=500$ on.

Our computations in these small graphs illustrate that $\max _{\tau} r_{T}$ decreases with $N$ and with the average degree (or the total number of links $L$ ). Figure 4 shows the typical bell shape of $r_{T}$ versus $\tau$ in connected Erdős-Rényi random graphs $G_{r}(N, L)$ for different number $L$ of links (or average degree $\left.d_{\mathrm{av}}=\frac{2 L}{N}\right)$. Similar bell shapes were found up to $N=14$. The maximum $\max _{\tau} r_{T}$ versus $N$ is plotted in Fig. 5 and indicates that $\max _{\tau} r_{T}$ decreases with $N$. The increase in accuracy (decrease of $\max _{\tau} r_{T}$ ) with both increasing average degree $d_{\text {av }}$ and network size $N$ agrees with the quality conditions for the Central Limit Theorem, as explained in Sec. II.

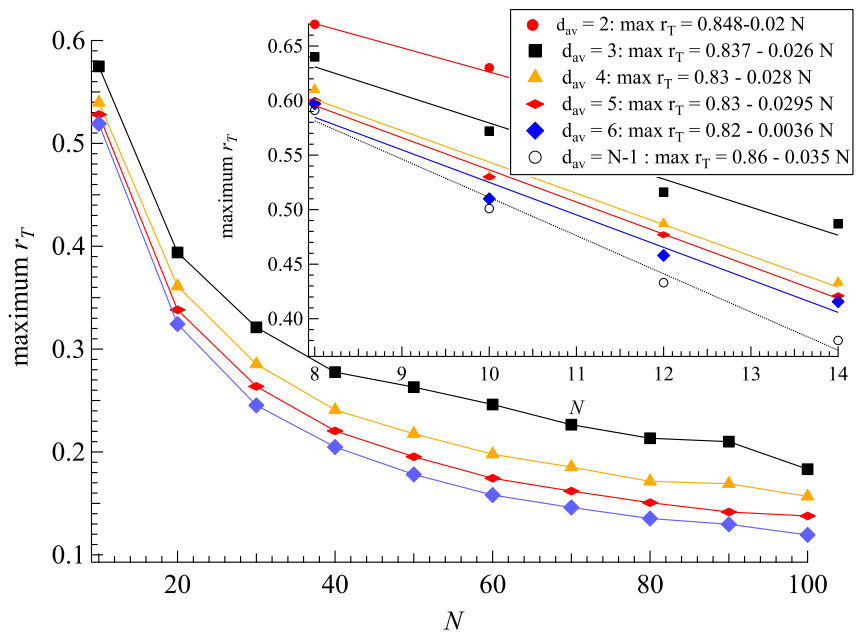

FIG. 5. (Color online) The maximum normalized topological MFA criterion $\max _{\tau} r_{T}$ vs the network size $N$ for connected ErdősRényi (ER) random graphs $G_{r}(N, L)$ with different average degree $d_{\mathrm{av}}=\frac{2 L}{N}$. Inset: Solutions of the Markov chain (for small $N$ ). The main plot was obtained after long simulations. For each $N$, a single realization of an ER graph was constructed on which the SIS process was simulated, which explains the lack of smoothness in $\max r_{T}$. 
Figures 3 and 5 lead us to hypothesize that for large $N$, in any graph, the worst MFA accuracy scales as

$$
\max _{\tau} r_{T}=O\left[\frac{1}{\lambda_{1}(A)}\right] \text {. }
$$

We support the conjecture by an approximate argument. Combining (7) and (8) and the definition (10) of $r_{T}$ shows that

$$
r_{T} \leqslant \frac{E_{G}}{L}\left[\lambda_{1}(C)-\lambda_{N}(C)\right] .
$$

Using [28], the graph energy $E_{G}=\sum_{j=1}^{N}\left|\lambda_{j}(A)\right|$ and $2 L=$ $\sum_{j=1}^{N} \lambda_{j}^{2}(A)$ yields, for large $N$,

$$
\frac{E_{G}}{L}=\frac{2 \sum_{j=1}^{N}\left|\lambda_{j}(A)\right|}{\sum_{j=1}^{N} \lambda_{j}^{2}(A)}=\frac{2}{\lambda_{1}(A)} \frac{1+\sum_{j=2}^{N} \frac{\left|\lambda_{j}(A)\right|}{\lambda_{1}}}{1+\sum_{j=2}^{N} \frac{\lambda_{j}^{2}(A)}{\lambda_{1}^{2}(A)}},
$$

and thus

$$
\max _{\tau} r_{T} \lesssim \frac{\xi(N)}{\lambda_{1}(A)}
$$

where

$$
\xi(N)=2 \lambda_{1}(C) \frac{1+\sum_{j=2}^{N} \frac{\left|\lambda_{j}(A)\right|}{\lambda_{1}}}{1+\sum_{j=2}^{N} \frac{\lambda_{j}^{2}(A)}{\lambda_{1}^{2}(A)}}
$$

Figure 3 seems to suggest that $\xi(N)=O(1)$ for $N \rightarrow \infty$, which leads to the conjecture (15). Extensive simulations or an analytic proof are needed to verify this claim. Moreover, it is worthwhile to explore for which other members of the LrGep class (in particular, the Kuramoto model as analyzed in [31]) such a type of scaling would apply.

\section{CONCLUSION}

The analysis of a MFA accuracy criterion for Markovian SIS epidemics on networks leads us to propose the normalized graph topology criterion $r_{T}$ in (10), which basically requires an evaluation of the spectral width $\lambda_{1}(C)-\lambda_{N}(C)$ of the covariance matrix $C$ of the SIS Markovian process and the graph energy $E_{G}$. The normalized graph topology criterion $r_{T}$ exhibits a bell-shaped curve as a function of the effective infection rate $\tau$, with a maximum at a slightly smaller $\tau$ value than $\tau^{*}$ for which the metastable state fraction of infected nodes equals one-half (i.e., $y_{\infty}\left(\tau^{*}\right)=\frac{1}{2}$ ). Computations on small graphs $(N \leqslant 14)$ reveal that the worst accuracy of MFA, measured by $\max _{\tau} r_{T}$, decreases with average degree $d_{\mathrm{av}}$ as well as with increasing size $N$ of the network, and these observations are in line with the applicability of the Central Limit Theorem.

Unfortunately, the numerical computation of the covariance matrix $C$ is difficult: the complete $2^{N} \times 2^{N}$ SIS Markov infinitesimal generator needs to be constructed and solved for the $\left(2^{N} \times 1\right)$-state vector (in the metastable state), which can only be executed for small $N$. Initial simulations (up to $N=100$ ) reveal the behavior of $\max _{\tau} r_{T}$ versus network size $N$ for the complete graph, star graph, square lattice, and path graph, and agree with MFA accuracy conditions derived from the Central Limit Theorem. Moreover, the calculations and simulations led us to conjecture the worst MFA accuracy scaling law in (15).

The extension of the analysis to larger and more realistic sizes of networks will require very long simulations. Approximate, higher-order mean-field computations, such as [26], are believed, in general, to be insufficiently accurate to determine $r_{T}$. At last, the analysis is specifically geared towards Markovian SIS epidemics and does not seem to be straightforwardly generalized to other LrGep models.

\section{ACKNOWLEDGMENTS}

We are very grateful to Claudio Castellano for helpful comments. This work has been partially supported by the European Commission within the framework of CONGAS (Project No. FP7-ICT-2011-8-317672) and the EU Network of Excellence EINS (Grant No. FP7-ICT 288021).

\section{APPENDIX A: UPPER BOUNDS FOR (7)}

Applying Abel's partial summation

$$
\sum_{k=1}^{n} a_{k} b_{k}=\sum_{k=1}^{n-1}\left(\sum_{l=1}^{k} a_{l}\right)\left(b_{k}-b_{k+1}\right)+b_{n}\left(\sum_{l=1}^{n} a_{l}\right)
$$

to (7) yields, with $\sum_{k=1}^{N} \lambda_{k}(A)=0$,

$$
\sum_{k=1}^{N} \lambda_{k}(A) \lambda_{k}(C)=\sum_{k=1}^{N-1}\left[\sum_{j=1}^{k} \lambda_{j}(A)\right]\left[\lambda_{k}(C)-\lambda_{k+1}(C)\right] .
$$

Since eigenvalues are implicitly assumed to be ordered, $\lambda_{1} \geqslant$ $\lambda_{2} \geqslant \cdots \geqslant \lambda_{N}$, the spacings $\lambda_{k}-\lambda_{k+1} \geqslant 0$, which illustrates that all terms in the above sums are non-negative because $\sum_{j=1}^{k} \lambda_{j}(A)>0$, for $1 \leqslant k<N$, and $\sum_{j=1}^{k} \lambda_{j}(A)=0$, only if $k=N$. If $l$ is the index for which $\lambda_{l}(A) \geqslant 0$ and $\lambda_{l+1}(A)<$ 0 , then $\sum_{j=1}^{k} \lambda_{l}(A)$ is increasing for $1 \leqslant k \leqslant l$ and decreasing for $l+1 \leqslant k \leqslant N$. The graph energy [28, p. 201], defined as $E_{G}=\sum_{j=1}^{N}\left|\lambda_{j}(A)\right|$, can be written as

$$
E_{G}=\sum_{j=1}^{l} \lambda_{j}(A)-\sum_{j=l+1}^{N}\left|\lambda_{j}(A)\right|=2 \sum_{j=1}^{l} \lambda_{j}(A)
$$

because $\operatorname{tr}(A)=0$ leads to $\sum_{j=1}^{l} \lambda_{j}(A)=-\sum_{j=l+1}^{N} \lambda_{j}(A)$. Hence, an upper bound for (A2) is

$$
\begin{aligned}
\sum_{k=1}^{N} \lambda_{k}(A) \lambda_{k}(C) & \leqslant \frac{E_{G}}{2} \sum_{k=1}^{N-1}\left[\lambda_{k}(C)-\lambda_{k+1}(C)\right] \\
& =\frac{E_{G}}{2}\left[\lambda_{1}(C)-\lambda_{N}(C)\right],
\end{aligned}
$$

while a lower bound of the same form $\left(\frac{E_{G}}{2} \times\right.$ a spacing between eigenvalues) is

$$
\begin{aligned}
\sum_{k=1}^{N} \lambda_{k}(A) \lambda_{k}(C) & >\left[\sum_{j=1}^{l} \lambda_{j}(A)\right]\left[\lambda_{l}(C)-\lambda_{l+1}(C)\right] \\
& =\frac{E_{G}}{2}\left[\lambda_{l}(C)-\lambda_{l+1}(C)\right] .
\end{aligned}
$$


The minimization of $\operatorname{tr}(A C)$ requires a low graph energy $E_{G} \geqslant$ $\sqrt{2 L}$, apart from a small eigenvalue spacing $\lambda_{1}(C)-\lambda_{N}(C)$.

\section{APPENDIX B: SPECTRUM OF C}

We analyze the spectrum of the Markovian SIS covariance matrix $C$ in a graph $G$, at any time $t$ and for any effective infection rate $\tau$. Any covariance matrix is positive semidefinite $[14$, p. 71$]$. Here, in addition, $C$ is a non-negative matrix with $i$ th diagonal element $c_{i i}=\operatorname{var}\left[X_{i}\right]=\sigma_{X_{i}}^{2}=E\left[X_{i}\right](1-$ $\left.E\left[X_{i}\right]\right)=w_{i}\left(1-w_{i}\right) \leqslant \frac{1}{4}$, because $X_{i}^{2}=X_{i}$ for Bernoulli random variables, from which

$$
\sum_{k=1}^{N} \lambda_{k}(C)=\operatorname{tr}(C)=\sum_{k=1}^{N} \operatorname{var}\left[X_{k}\right] \leqslant \frac{N}{4} .
$$

By the Cauchy-Schwarz inequality [14]

$$
\begin{aligned}
c_{i j} & =\operatorname{cov}\left[X_{i}, X_{j}\right]=E\left\{\left(X_{i}-E\left[X_{i}\right]\right)\left(X_{j}-E\left[X_{j}\right]\right)\right\} \\
& \leqslant \sqrt{E\left\{\left(X_{i}-E\left[X_{i}\right]\right)^{2}\right\}} \sqrt{E\left\{\left(X_{j}-E\left[X_{j}\right]\right)^{2}\right\}}=\sigma_{X_{i}} \sigma_{X_{j}},
\end{aligned}
$$

we obtain that $c_{i j} \leqslant \sigma_{X_{i}} \sigma_{X_{j}}$, with equality on the diagonal, $c_{i i}=\sigma_{X_{i}}^{2}$. The symmetric matrix $\widetilde{C}$, with element $\widetilde{c}_{i j}=$ $\sigma_{X_{i}} \sigma_{X_{j}}$, has the same diagonal elements as $C$, but larger off-diagonal elements than $C$. For Markovian SIS and large $N$, the variance $\operatorname{var}\left[X_{k}\right]$ is shown in [37] to be largest for effective infection rates $\tau$ around, but above, the epidemic threshold $\tau_{c}$ (see also Figs. 1 and 2 for the complete graph and star graph). Furthermore, $\widetilde{C}=z z^{T}$, where the vector $z=$ $\left(\sigma_{X_{1}}, \sigma_{X_{2}}, \ldots, \sigma_{X_{N}}\right)$ and $x^{T} \widetilde{C} x=\left(x^{T} z\right)^{2} \geqslant 0$ shows that just like $C, \widetilde{C}$ is also positive semidefinite. Since $\widetilde{C}$ is a rank-one matrix, the eigenvalues are $\lambda_{k}(\widetilde{C})=0$ (for $1<k \leqslant N$ ) and $\lambda_{1}(\widetilde{C})=z^{T} z=\sum_{k=1}^{N} \operatorname{var}\left[X_{k}\right]$. Hence, the spectrum of $\widetilde{C}$ has a large gap with a clearly dominant largest eigenvalue. Since the matrix $\widetilde{C}$ "dominates" the matrix $C$ elementwise and $\operatorname{tr}(A C) \leqslant$ $\operatorname{tr}(A \widetilde{C}),\|R\|_{1} \rightarrow 0$ for $\tau<\tau_{c}$ and $\tau \rightarrow \infty$ because, in that region and limit, any variance $\sigma_{X_{j}}^{2}=w_{i}\left(1-w_{i}\right)$ vanishes. We will now show that the $C$ matrix inherits the spectral properties of $\widetilde{C}$, though in a less sharp way.

Let us consider the symmetric matrix $\Gamma=\widetilde{C}-C$, which is a non-negative matrix. By the Perron-Frobenius theorem of non-negative matrices, the largest eigenvalue $\lambda_{1}(\Gamma)$ is positive and Gerschgorin's theorem shows that $\lambda_{1}(\Gamma) \leqslant \max _{i}$ $\sum_{j=1}^{N}\left(\sigma_{X_{i}} \sigma_{X_{j}}-c_{i j}\right)$. The Perron-Frobenius theorem also tells us that $-\lambda_{N}(\Gamma) \leqslant \lambda_{1}(\Gamma)$. Since $\operatorname{tr}(\Gamma)=0$, at least one eigenvalue is negative and, thus, $\Gamma$ is not positive semidefinite. Now, the eigenvalues of $C$ are the zeros of the characteristic polynomial

$$
\begin{aligned}
\operatorname{det}(C-\xi I) & =\operatorname{det}(\widetilde{C}-\Gamma-\xi I)=\operatorname{det}\left[z z^{T}-(\Gamma+\xi I)\right] \\
& =(-1)^{N} \operatorname{det}\left[(\Gamma+\xi I)-z z^{T}\right] \\
& =(-1)^{N} \operatorname{det}(\Gamma+\xi I)\left[1-z^{T}(\Gamma+\xi I)^{-1} z\right],
\end{aligned}
$$

where, in the last line, the general rank-one update formula [28],

$$
\operatorname{det}\left(A_{n \times n}+C_{n \times k} D_{k \times n}^{T}\right)=\operatorname{det} A \operatorname{det}\left(I_{k}+D^{T} A^{-1} C\right),
$$

has been used for $k=1$. Invoking the resolvent of a symmetric matrix

$$
(\Gamma+\xi I)^{-1}=\sum_{j=1}^{N} \frac{1}{\lambda_{j}(\Gamma)+\xi} x_{j}(\Gamma) x_{j}^{T}(\Gamma),
$$

where $x_{j}(\Gamma)$ is the eigenvector of $\Gamma$, normalized as $x_{j}^{T}(\Gamma) x_{j}(\Gamma)=1$ and belonging to the eigenvalue $\lambda_{j}(\Gamma)$, leads to

$$
\operatorname{det}(C-\xi I)=(-1)^{N} \operatorname{det}(\Gamma+\xi I)\left\{1-\sum_{j=1}^{N} \frac{\left[z^{T} x_{j}(\Gamma)\right]^{2}}{\lambda_{j}(\Gamma)+\xi}\right\},
$$

which illustrates, with $(-1)^{N} \operatorname{det}(\Gamma+\xi I)=\prod_{j=1}^{N}[\xi+$ $\left.\lambda_{j}(\Gamma)\right]$ (see [28, p. 248]), that the eigenvalues of $C$ (except those with multiplicity larger than 1) are all different from those of $\Gamma$ and obey

$$
\sum_{j=1}^{N} \frac{\left[z^{T} x_{j}(\Gamma)\right]^{2}}{\lambda_{j}(\Gamma)+\xi}=1
$$

This partial fraction expansion (B1) is decreasing in $\xi$ and has poles at $\xi=-\lambda_{j}(\Gamma)$ so that all $\lambda_{j}(C)$ interlace with and are close to those of $\lambda_{j}(\Gamma)$,

$$
\begin{aligned}
-\lambda_{1}(\Gamma) & <\lambda_{N}(C) \leqslant-\lambda_{2}(\Gamma) \\
& \leqslant \lambda_{N-1}(C) \leqslant \cdots \leqslant-\lambda_{N}(\Gamma) \leqslant \lambda_{1}(C) .
\end{aligned}
$$

Since $C$ is positive semidefinite, all eigenvalues of $\lambda_{j}(\Gamma)$, except for $\lambda_{1}(\Gamma)$, must be negative. Thus, $\lambda_{1}(\Gamma)=-\sum_{k=2}^{N} \lambda_{k}(\Gamma)$ because $\operatorname{tr}(\Gamma)=0$.

For large negative (positive) $\xi$, all terms of the sum in (B1) are negative (positive). Just above the pole at $-\lambda_{1}(\Gamma)$, i.e., $\xi>-\lambda_{1}(\Gamma)$, the term $\frac{\left(z^{T} x_{1}(\Gamma)\right)^{2}}{\lambda_{1}(\Gamma)+\xi}>0$ dominates the sum in (B1), so that the sign reverses from negative to positive. Since there cannot be a negative eigenvalue of $C$, there must hold at $\xi=0$ that

$$
\sum_{j=1}^{N} \frac{\left[z^{T} x_{j}(\Gamma)\right]^{2}}{\lambda_{j}(\Gamma)} \geqslant 1
$$

from which

$$
\begin{aligned}
\lambda_{1}(\Gamma) \leqslant & \frac{\left[z^{T} x_{1}(\Gamma)\right]^{2}}{1-\sum_{j=2}^{N} \frac{\left[z^{T} x_{j}(\Gamma)\right]^{2}}{\lambda_{j}(\Gamma)}}<\left[z^{T} x_{1}(\Gamma)\right]^{2} \\
& =\left\{\sum_{m=1}^{N} \sigma_{X_{m}}\left[x_{1}(\Gamma)\right]_{m}\right\}^{2},
\end{aligned}
$$

where $\left[x_{1}(\Gamma)\right]_{m}>0$ (by the Perron-Frobenius theorem). The Cauchy-Schwarz inequality shows that

$$
\begin{aligned}
\left\{\sum_{m=1}^{N} \sigma_{X_{m}}\left[x_{1}(\Gamma)\right]_{m}\right\}^{2} & \leqslant \sum_{m=1}^{N} \sigma_{X_{m}}^{2} \sum_{m=1}^{N}\left[x_{1}(\Gamma)\right]_{m}^{2} \\
& =\sum_{m=1}^{N} \sigma_{X_{m}}^{2}=\lambda_{1}(\widetilde{C}),
\end{aligned}
$$

and thus $\lambda_{1}(\widetilde{C})>\lambda_{1}(\Gamma)$. The largest eigenvalue of $C$ is larger than $-\lambda_{N}(\Gamma)$. Indeed, consider $\xi=\lambda_{1}(C) \geqslant-\lambda_{N}(\Gamma)$; then 
the partial fraction expansion (B1) shows that

$$
-\lambda_{N}(\Gamma)+\frac{\left[z^{T} x_{N}(\Gamma)\right]^{2}}{1-\sum_{j=1}^{N-1} \frac{\left[z^{T} x_{j}(\Gamma)\right]^{2}}{\lambda_{j}(\Gamma)+\lambda_{1}(C)}}=\lambda_{1}(C),
$$

which allows us to compute $\lambda_{1}(C)$ (recursively as in [28, p. 138]) in terms of the eigenvalues of $\Gamma$. Also, the spectral gap $\lambda_{1}(C)-\lambda_{2}(C)>\lambda_{1}(C)+\lambda_{N}(\Gamma)$.

Since

$$
\lambda_{1}(C)=\sum_{k=1}^{N} \operatorname{var}\left[X_{k}\right]-\sum_{k=2}^{N} \lambda_{k}(C)
$$

and the interlacing of the "band eigenvalues" causes $\sum_{k=2}^{N} \lambda_{k}(C) \approx-\sum_{k=2}^{N} \lambda_{k}(\Gamma)=\lambda_{1}(\Gamma)$, we see that, approximately,

$$
\lambda_{1}(C) \approx \sum_{k=1}^{N} \sigma_{X_{k}}^{2}-\lambda_{1}(\Gamma)=\lambda_{1}(\widetilde{C})-\lambda_{1}(\Gamma),
$$

which points to the fact that both $\Gamma$ and $C$ have a large spectral gap. ${ }^{5}$

Thus, the sharp spectrum of $\widetilde{C}$ is smoothed: the spectral gap of $C$ is smaller and the other eigenvalues of $C$ lie in a tiny "band," slightly above zero rather than collapsed at zero for $\widetilde{C}$.

\section{APPENDIX C: EIGENVALUES OF $C_{\text {star }}$ FOR THE STAR GRAPH}

Let the center of the star graph be labeled by 1, while the leaf nodes range from 2 up to $N$. Due to symmetry, the metastable state covariance matrix $C_{\text {star }}$ for the star graph has only four different values: $c_{11}=a, c_{j j}=b$ and $c_{1 j}=c$ for $j>1, c_{i j}=d$ for $i>1$ and $j>1$. Hence, the structure is

$$
C_{\mathrm{star}}=\left[\begin{array}{ccccc}
a & c & c & \cdots & c \\
c & b & d & \cdots & d \\
c & d & b & \cdots & d \\
\vdots & \vdots & & \ddots & \vdots \\
c & d & d & \cdots & b
\end{array}\right] .
$$

The determinant $\operatorname{det}\left(C_{\text {star }}-\lambda I\right)$ is evaluated using Schur's complement formula [28],

$$
\operatorname{det}\left[\begin{array}{ll}
A & B \\
C & D
\end{array}\right]=\operatorname{det} A \operatorname{det}\left(D-C A^{-1} B\right),
$$

where

$$
\begin{aligned}
A & =\left[\begin{array}{cc}
a-\lambda & c \\
c & b-\lambda
\end{array}\right] \text { and } A^{-1}=\frac{1}{(a-\lambda)(b-\lambda)-c^{2}}\left[\begin{array}{cc}
b-\lambda & -c \\
-c & a-\lambda
\end{array}\right], \\
B_{2 \times(N-2)} & =\left[\begin{array}{lll}
c & \cdots & c \\
d & \cdots & d
\end{array}\right]=C^{T},
\end{aligned}
$$

and $D=d J_{(N-2) \times(N-2)}+(b-d-\lambda) I_{N-2}$. Hence,

$$
C A^{-1} B=\frac{1}{(a-\lambda)(b-\lambda)-c^{2}}\left[\begin{array}{cc}
c & d \\
\vdots & \vdots \\
c & d
\end{array}\right]\left[\begin{array}{cc}
b-\lambda & -c \\
-c & a-\lambda
\end{array}\right]\left[\begin{array}{ccc}
c & \cdots & c \\
d & \cdots & d
\end{array}\right]=\frac{c^{2}(b-\lambda)+d^{2}(a-\lambda)-2 c^{2} d}{(a-\lambda)(b-\lambda)-c^{2}} J_{(N-2) \times(N-2)}
$$

and

$$
\begin{aligned}
D-C A^{-1} B & =\left[d-\frac{c^{2}(b-\lambda)+d^{2}(a-\lambda)-2 c^{2} d}{(a-\lambda)(b-\lambda)-c^{2}}\right] J_{(N-2) \times(N-2)}+(b-d-\lambda) I_{N-2} \\
& =\frac{d(a-\lambda)(b-\lambda)-c^{2}(b-\lambda)-d^{2}(a-\lambda)+c^{2} d}{(a-\lambda)(b-\lambda)-c^{2}} u u^{T}+(b-d-\lambda) I .
\end{aligned}
$$

Next,

$$
d(a-\lambda)(b-\lambda)-c^{2}(b-\lambda)-d^{2}(a-\lambda)+c^{2} d=\left[d(a-\lambda)-c^{2}\right](b-\lambda-d) .
$$

Further,

$\operatorname{det}\left(D-C A^{-1} B\right)=\operatorname{det}\left\{\frac{\left[d(a-\lambda)-c^{2}\right](b-d-\lambda)}{(a-\lambda)(b-\lambda)-c^{2}} u u^{T}+(b-d-\lambda) I\right\}=(b-d-\lambda)^{N-2} \operatorname{det}\left\{\frac{\left[d(a-\lambda)-c^{2}\right]}{(a-\lambda)(b-\lambda)-c^{2}} u u^{T}+I\right\}$.

${ }^{5}$ For large $N$, we may apply the Marcenko-Pastur law [28] for the spectrum of $C$. 
Using the rank-one update formula [28],

$$
\operatorname{det}\left(I+c d^{T}\right)=1+d^{T} c
$$

yields

$$
\begin{aligned}
\operatorname{det}\left(D-C A^{-1} B\right) & =(b-d-\lambda)^{N-2}\left\{1+\frac{\left[d(a-\lambda)-c^{2}\right]}{(a-\lambda)(b-\lambda)-c^{2}}(N-2)\right\} \\
& =(b-d-\lambda)^{N-2}\left\{\frac{(a-\lambda)(b-\lambda)-c^{2}+(N-2)\left[d(a-\lambda)-c^{2}\right]}{(a-\lambda)(b-\lambda)-c^{2}}\right\}
\end{aligned}
$$

and

$$
\operatorname{det}\left(C_{\text {star }}-\lambda I\right)=(b-d-\lambda)^{N-2}\left[(a-\lambda)(b-\lambda)+(N-2) d(a-\lambda)-(N-1) c^{2}\right] .
$$

The quadratic polynomial ${ }^{6}$ is

$$
p_{2}(\lambda)=(a-\lambda)(b-\lambda)+(N-2) d(a-\lambda)-(N-1) c^{2}=\lambda^{2}-\{(a+b)+(N-2) d\} \lambda+a b+(N-2) d a-(N-1) c^{2},
$$

with roots

$$
2 \lambda_{1}=(a+b)+(N-2) d \pm \sqrt{[(a+b)+(N-2) d]^{2}-4\left[a b+(N-2) d a-(N-1) c^{2}\right]} .
$$

The discriminant is

$$
D=[(a+b)+(N-2) d]^{2}-4\left[a b+(N-2) d a-(N-1) c^{2}\right]=[b-a+(N-2) d]^{2}+4(N-1) c^{2} .
$$

Finally, the eigenvalues of $C_{\text {star }}$ are $b-d$ with multiplicity $N-2$ and

$$
\begin{aligned}
2 \lambda_{1}\left(C_{\text {star }}\right)= & (a+b)+(N-2) d \\
& +\sqrt{\left\{[b-a+(N-2) d]^{2}+4(N-1) c^{2}\right\}}
\end{aligned}
$$

and

$$
\begin{aligned}
2 \lambda_{N}\left(C_{\text {star }}\right)= & (a+b)+(N-2) d \\
& -\sqrt{\left\{[b-a+(N-2) d]^{2}+4(N-1) c^{2}\right\}} .
\end{aligned}
$$

\footnotetext{
${ }^{6}$ We may verify that the sum of the eigenvalues equals

$\operatorname{tr}\left(C_{\text {star }}\right)=a+(N-1) b=(N-2)(b-d)+(a+b)+(N-2) d$.
}

Since all eigenvalues must be non-negative, we find from $\lambda_{N}\left(C_{\text {star }}\right) \geqslant 0$ that

$$
\begin{aligned}
& (a+b)+(N-2) d \\
& \geqslant \sqrt{\left\{[b-a+(N-2) d]^{2}+4(N-1) c^{2}\right\}},
\end{aligned}
$$

which leads to a bound for

$$
c^{2} \leqslant a\left(\frac{b+d}{N-1}+d\right) .
$$

Thus, for large $N$, we find that $c^{2} \leqslant a d$.
[1] R. Pastor-Satorras, C. Castellano, P. Van Mieghem, and A. Vespignani, Epidemic processes in complex networks, arXiv:1408.2701 [Rev. Mod. Phys. (to be published)].

[2] L. Onsager, Crystal statistics: A two-dimensional model with an order-disorder transition, Phys. Rev. 65, 117 (1944).

[3] S. H. Strogatz, From Kuramoto to Crawford: Exploring the onset of synchronization in populations of coupled oscillators, Physica D 143, 1 (2000).

[4] P. Bak, C. Tang, and K. Wiesenfeld, Self-organized criticality: An explanation of 1/f noise, Phys. Rev. Lett. 59, 381 (1987).

[5] K.-I. Goh, D.-S. Lee, B. Kahng, and D. Kim, Sand pile on scale-free networks, Phys. Rev. Lett. 91, 148701 (2003).

[6] T. Kleiberg and P. Van Mieghem, Self-Organization of Internet Paths, in IFIP Fourth International Workshop on SelfOrganizing Systems 2009, Lecture Notes in Computer Science Vol. 5918 (Springer, Berlin, 2009), pp. 77-88.
[7] C. Castellano, S. Fortunato, and V. Loreto, Statistical physics of social dynamics, Rev. Mod. Phys. 81, 591 (2009).

[8] Q. Li, L. A. Braunstein, H. Wang, J. Shao, H. E. Stanley, and S. Havlin, Nonconsensus opinion models on complex networks, J. Stat. Phys. 151, 92 (2013).

[9] H. E. Stanley, Introduction to Phase Transitions and Critical Phenomena (Oxford University Press, Oxford, 1987).

[10] N. T. J. Bailey, The Mathematical Theory of Infectious Diseases and its Applications, 2nd ed. (Charlin Griffin, London, 1975).

[11] R. M. Anderson and R. M. May, Infectious Diseases of Humans: Dynamics and Control (Oxford University Press, Oxford, 1991).

[12] D. J. Daley and J. Gani, Epidemic Modeling: An Introduction (Cambridge University Press, Cambridge, 1999).

[13] O. Diekmann, H. Heesterbeek, and T. Britton, Mathematical Tools for Understanding Infectious Disease Dynamics (Princeton University Press, Princeton, NJ, 2012). 
[14] P. Van Mieghem, Performance Analysis of Complex Networks and Systems (Cambridge University Press, Cambridge, 2014).

[15] P. Van Mieghem, J. Omic, and R. E. Kooij, Virus spread in networks, IEEE/ACM Trans. Networking 17, 1 (2009).

[16] P. Van Mieghem, The $N$-Intertwined SIS epidemic network model, Computing 93, 147 (2011).

[17] P. Van Mieghem and E. Cator, Epidemics in networks with nodal self-infections and the epidemic threshold, Phys. Rev. E 86, 016116 (2012).

[18] E. Cator and P. Van Mieghem, Susceptible-infected-susceptible epidemics on the complete graph and the star graph: Exact analysis, Phys. Rev. E 87, 012811 (2013).

[19] A. Economou, A. Gómez-Corral, and M. López-García, A stochastic SIS epidemic model with heterogeneous contacts, Physica A 421, 78 (2015).

[20] R. J. Baxter, Exactly Solved Models in Statistical Mechanics (Academic, London, 1982).

[21] A. L. Fetter and J. D. Walecka, Quantum Theory of Manyparticle Systems (McGraw-Hill, San Francisco, 1971).

[22] P. A. M. Dirac, The Principles of Quantum Mechanics, 4th ed. (Clarendon, Oxford, 1986).

[23] C. Cohen-Tannoudji, B. Diu, and F. Laloë, Mécanique Quantique (Hermann, Paris, 1977), Vols. I and II.

[24] W. Feller, An Introduction to Probability Theory and Its Applications, 2nd ed. (Wiley, New York, 1971), Vol. 2.

[25] C. Li, R. van de Bovenkamp, and P. Van Mieghem, Susceptibleinfected-susceptible model: A comparison of $N$-intertwined and heterogeneous mean-field approximations, Phys. Rev. E 86, 026116 (2012).

[26] E. Cator and P. Van Mieghem, Second order mean-field SIS epidemic threshold, Phys. Rev. E 85, 056111 (2012).
[27] E. Cator and P. Van Mieghem, Nodal infection in Markovian SIS and SIR epidemics on networks are non-negatively correlated, Phys. Rev. E 89, 052802 (2014).

[28] P. Van Mieghem, Graph Spectra for Complex Networks (Cambridge University Press, Cambridge, 2011).

[29] J. Als-Nielsen and R. J. Birgenau, Mean field theory, the Ginzbug criterion, and marginal dimensionality of phase transitions, Am. J. Phys. 45, 554 (1977).

[30] P. Van Mieghem and R. van de Bovenkamp, Non-Markovian infection spread dramatically alters the SIS epidemic threshold in networks, Phys. Rev. Lett. 110, 108701 (2013).

[31] P. Van Mieghem, Epidemic phase transition of the SIS-type in networks, Europhys. Lett. 97, 48004 (2012).

[32] P. Van Mieghem, The viral conductance of a network, Comput. Commun. 35, 1494 (2012).

[33] R. van de Bovenkamp and P. Van Mieghem, Survival time of the SIS infection process on a graph [Phys. Rev. E (to be published)].

[34] R. E. Kooij, P. Schumm, C. Scoglio, and M. Youssef, A new metric for robustness with respect to virus spread, in Networking 2009, Lecture Notes in Computer Science Vol. 5550 (Springer, Heidelberg, 2009), p. 562.

[35] M. Youssef, R. E. Kooij, and C. Scoglio, Viral conductance: Quantifying the robustness of networks with respect to spread of epidemics, J. Comput. Sci. 2, 286 (2011).

[36] J. Martin Hernandez, T. Kleiberg, H. Wang, and P. Van Mieghem, International Symposium on Performance Evaluation of Computer and Telecommunication Systems (SPECTS 2007) (IEEE, New York, 2007).

[37] P. Van Mieghem, Exact Markovian SIR and SIS epidemics on networks and an upper bound for the epidemic threshold, arXiv:1402.1731. 